> Qu'il s'agisse de microscopes ou d'appareils photo, de jumelles, de longues-vues ou de télescopes, les firmes Zeiss et Leitz sont parmi les plus connues au monde en matière d'optique. Nées toutes deux au milieu du $X I X^{e}$ siècle, avant les compagnies nippones Nikon et Canon, elles ont réussi à traverser, chacune à leur manière, les ébranlements des Première et Seconde Guerres mondiales et les vicissitudes de la partition puis de la réunification de l'Allemagne. Sur leur histoire, les documents d'archives ne manquent pas, mais méritent une analyse attentive pour mieux comprendre ou deviner le rôle des principaux acteurs et leurs contributions aux progrès de l'industrie optique. L'histoire de l'entreprise Zeiss présentée ici sera suivie par l'histoire de la maison Leitz puis par celle d'Emmanuel Goldberg, dans les prochains numéros de médecine/sciences. <

\title{
Grandeur \\ et vicissitudes \\ de deux grandes \\ entreprises \\ d'optique \\ allemandes
}

\author{
I. La firme Zeiss
}

Simone Gilgenkrantz

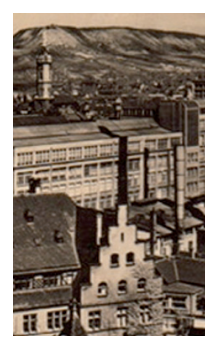

S. Gilgenkrantz :

9 , rue Basse,

54330 Clérey-sur-Brénon, France.

simsimone.gilgenkrantz@gmail.com

\section{L’entreprise Zeiss}

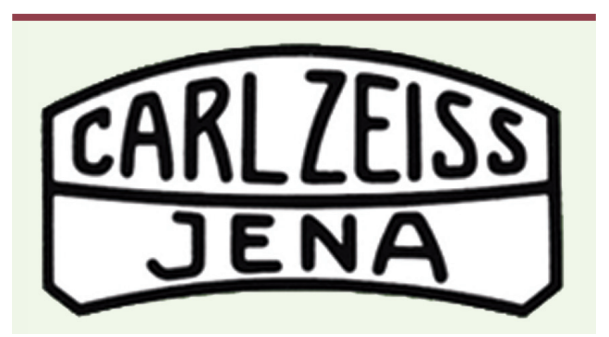

Logo de Zeiss jusqu'en 1945

La sagesse du père fondateur

Carl Friedrich Zeiss (1816-1888) est le cinquième enfant d'une fratrie de douze, dont beaucoup décéderont en bas âge. Il est issu d'un milieu aisé. Sa mère appartient à une famille de juristes. Son père est fabricant d'objets d'art à Weimar et compte dans sa clientèle le grand duc de Saxe-Weimar-Eisenach. Carl Zeiss s'oriente très tôt vers les sciences naturelles et la mécanique. II se forme auprès de Friedrich Körner (concepteur d'instruments scientifiques) et à l'université d'léna où il suit des cours de mathématiques, de physique, de minéralogie et d'optique.

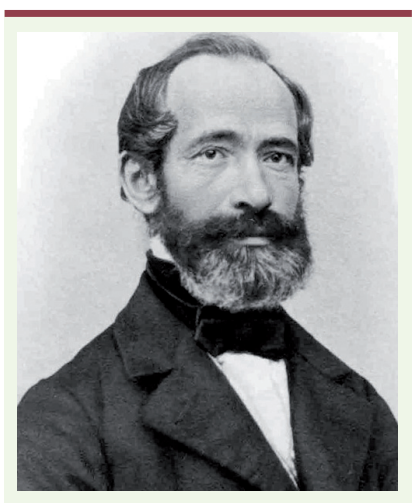

Carl Friedrich Zeiss (1816-1888)

En 1846, il obtient l'autorisation d'ouvrir à léna une fabrique de matériel de mécanique et d'optique de précision. Dès la première année, il vend plus de vingt microscopes classiques et embauche son premier employé. Puis, il conçoit un microscope doté d'un objectif et d'un oculaire : le Stand I. En 1861, il obtient une médaille d'or à la foire industrielle de Thuringe. En 1866, il a déjà vendu son millième microscope et sa renommée s'étend dans les milieux scientifiques européens. La même année, il décide de recruter un jeune physicien de 26 ans, enseignant à l'université d'léna, Ernst Abbe, remarquablement doué. Ce dernier révolutionne la fabrication des lentilles 
qui repose désormais sur les lois physiques: «Abbe sin condition ». II définit «le nombre d'Abbe », ou constringence.

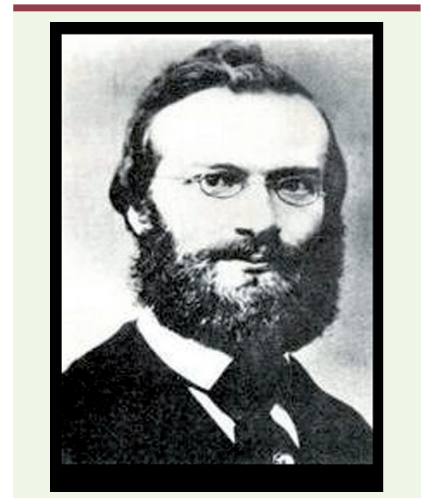

Ernst Abbe (1840-1905)

En 1881, Ernst Abbe entre en contact avec un verrier, Otto Schott (18511935), qui réussit à produire des verres dont la pureté et les qualités optiques sont inégalées. À 31 ans, Otto Schott quitte sa ville de Witten dans la Ruhr et installe en 1884 à léna un atelier de recherche sur le verre: Jenaer Glaswerke Schott \& Genossen, puis en crée un autre à Mainz: Schott \& Genossen Glaswerke, pour produire diverses variétés de verre. Ces recherches portent leurs fruits : pour la première fois, en 1886, est conçue une nouvelle catégorie d'objectifs : les apochromatiques (ou APO) qui corrigent les aberrations chromatiques des oculaires en immersion dans l'eau.

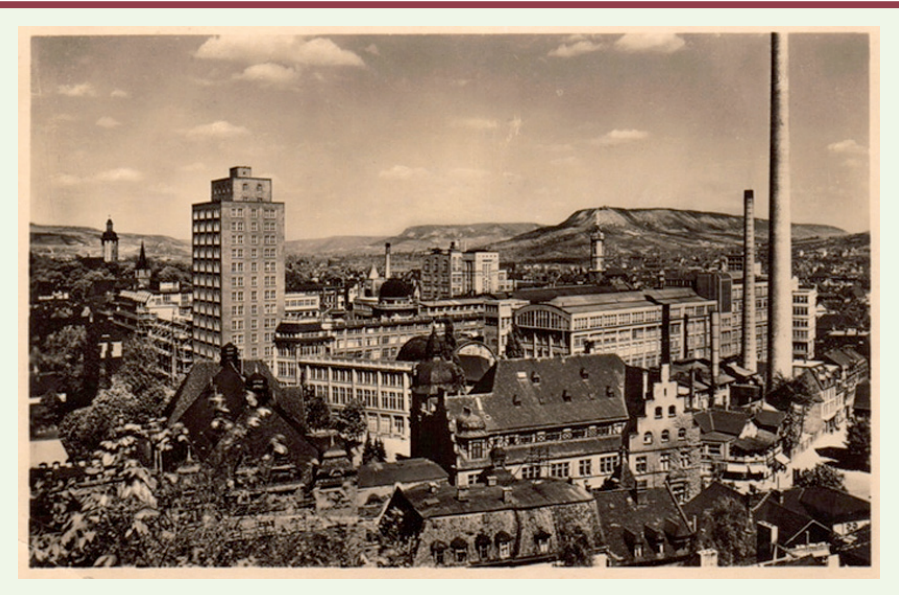

Carl Zeiss léna vers 1910

\section{La Carl-Zeiss-Stiftung}

La fabrique emploie alors 250 employés et a déjà vendu 10000 microscopes. Le fondateur, Carl Zeiss, vécut assez longtemps pour assister à cette réussite. Quand il meurt, en 1888, à l'âge de 72 ans, son fils Roderick $^{1}$ reprend l'affaire pour un temps très court car il devient

${ }^{1}$ Roderick était le fils unique de la première union de Carl, avec Bertha Schatter, de 11 ans plus jeune que son mari. Sa mère mourut à 23 ans des suites de couches. Carl se remaria trois ans plus tard avec 0ttilie Trinkler et eut trois autres enfants dont on ne retrouve pas trace dans l'histoire de l'entreprise. infirme à la suite d'un accident. Abbe, qui avait racheté des parts de l'entreprise décide alors de transformer la firme Zeiss en une fondation: la Carl-Zeiss-Stiftung, dont les statuts sont extrêmement novateurs pour l'époque. La direction est collégiale, assurée par des membres d'un conseil d'administration. Les employés désignent leurs représentants, participent aux profits, bénéficient d'une assurance maladie et d'une retraite. Cette structure originale a assuré à la fondation Zeiss une capacité d'adaptation et d'expansion qui perdure encore de nos jours.

Dans les premières années $d u x x^{e}$ siècle, des succursales et des usines satellites sont créées un peu partout en Europe (Londres au Royaume-Uni, Vienne en Autriche, Györ en Hongrie, Riga en Lettonie). La production se diversifie, entre autres dans le matériel militaire. Dès le début de la Première Guerre mondiale, Zeiss développe sa production de jumelles pour l'armée allemande. En dépit de la Grande Dépression qui suit la défaite et malgré une compression du personnel, la firme continue à se développer. Il est assez ironique d'observer que les succursales aux Pays-Bas (Venlo) ou en Russie (SaintPétersbourg) fabriqueront des jumelles et du matériel d'optique militaire sophistiqué que la Wehrmacht utilisera quand, plus tard, elle envahira ces pays. En 1925, une Carl Zeiss, Inc. s'installe à New York avec un directeur allemand, Karl Bauer. Aussi étonnant que cela puisse paraître, son activité commerciale se maintiendra pendant toute la Seconde Guerre mondiale.

Zeiss acquiert aussi des usines de production d'appareils photo à Berlin, à Stuttgart et à Dresde, qui fusionneront pour devenir Zeiss Ikon (1926). Mais le premier modèle de 24 x 36 télémétrique, le Contax, en 1932, ne réussit pas à supplanter le Leica conçu par la maison Leitz. Nous aurons l'occasion d'y revenir.

\section{L’obscure période nazie}

L'avènement du national socialisme fut une aubaine pour l'expansion des usines Zeiss, à léna et à Dresde. Parmi les grandes entreprises allemandes, la Carl-ZeissStiftung devint un fleuron du régime. Un commissaire, désigné par le parti, fut nommé en 1933, puis remplacé en 1934 par Abraham Esau, ancien doyen de l'université d'léna, qui devint par la suite un des hauts responsables du projet allemand d'énergie nucléaire [1]. Il est très difficile de connaître la position des responsables visà-vis du régime, dans cette atmosphère d'invincibilité régnant au début de la guerre. D'autant que l'optique Zeiss, désormais orientée vers le matériel de guerre, contribue largement à la qualité et à l'efficacité de l'équipement des armées de terre, de mer et de l'air: 
une des photos les plus publiées au début de la guerre montre Hitler observant au périscope binoculaire d'artillerie l'attaque de Varsovie par les tirs de l'armée, appuyée par la Luftwaffe.

Les employés juifs disparaissent, et pour compenser le départ de tous les Allemands mobilisés, de nombreux prisonniers de guerre, déportés (du camp de Flossenbourg) et du service de travail obligatoire (STO) les remplacent. Mais, bien que les données historiques sur la fondation Zeiss soient pléthoriques, en particulier avec la Zeiss Historica Society (ZHS) ${ }^{2}$ qui rend compte de l'histoire chronologique de la maison Zeiss, il existe peu de renseignements précis sur les événements qui se sont déroulés entre 1932 et 1945.

La ZHS fournit quelques biographies de ses principaux ingénieurs, comme celle de l'Autrichien Hubert Nerwin (1906-1983) qui créa le logo de Zeiss, utilisé jusqu'en 1945 , ou des responsables comme Heinz Küppenbender (1901-1989) qui fut d'abord directeur de Zeiss Ikon, puis de l'ensemble de la Carl-Zeiss-Stiftung jusqu'à sa retraite en 1972, à l'âge de 71 ans. Au moment de la défaite du Troisième Reich, après un bref procès en dénazification (il était membre du parti nazi), c'est lui qui est choisi par les autorités américaines pour procéder au transfert d'une partie des scientifiques et des techniciens vers l'Allemagne de l'Ouest à Oberkochen (dans le Land de Bade-Würtemberg). Mais l'histoire des employés et responsables chassés par les nazis reste floue et ce n'est que par des recherches patientes que I'on retrouve parmi ceux-ci les noms de Wandersleb, Schrabe, Boegehold, disparus de la firme à partir de 1933. Sans oublier Emanuel Goldberg, pionnier de la photographie, des microfilms [2, 3] et surtout de l'informatique [4], dont le nom fut délibérément effacé des archives de la maison Zeiss. Ce n'est qu'au XXI ${ }^{e}$ siècle que la contribution de ce génial inventeur fut redécouverte [5]. Nous lui consacrerons le $3^{e}$ article de cette série $(\rightarrow)$ afin de compléter l'histoire de la $(\rightarrow) \mathrm{m} / \mathrm{s}, 2011$ firme Zeiss pendant la période nazie. (sous presse)

\section{Défaite et démantèlement de la maison Zeiss}

Le 13 avril 1945, la troisième armée américaine atteint léna. Les combats ont laissé la fabrique presque intacte. Très vite, les directeurs, dont Walter Bauersfeld en poste depuis 1908 (il conservera des responsabilités jusqu'à sa mort à l'âge de 80 ans), Heinz Kuppenbender, ainsi que 130 ingénieurs et techniciens sont évacués par camion en Allemagne de l'Ouest, vers la zone d'occupation américaine car, selon les accords de Yalta (février 1945), la

${ }^{2}$ http://www.zeisshistorica.org/index.html. partie est de l'Allemagne devait être sous domination russe. L'installation se fait à Oberkochen, dans les locaux d'une usine désaffectée qui fabriquait des trains d'atterrissage pour la Luftwaffe. II fallait repartir à zéro car les équipements de laboratoire et le matériel qui devaient suivre (et dont certains refirent surface aux États-Unis) ne parvinrent jamais dans le Land de Bade-Würtemberg. Mais la plupart des chercheurs et des concepteurs, fuyant l'occupation russe, s'y retrouvaient, ainsi qu'une partie des universitaires d'léna. Dès 1946, la production de microscopes et autres matériels d'optique reprend et se diversifie au cours des décennies : en 1980, la compagnie Zeiss est représentée dans tous les continents et exporte $50 \%$ de sa production.

En juillet 1945, léna est occupée par les troupes soviétiques. Outre les appareils photo qui seront fabriqués à Kiev, tout ce qui peut servir à la fabrication de microscopes est envoyé à Leningrad (Saint-Pétersbourg) comme indemnités de guerre pour la manufacture LOMO ; des ingénieurs sont transférés en Union soviétique. Pourtant, dès juin 1948, un combinat Carl Zeiss se réorganise à léna sous le nom de VEB ${ }^{3}$ Carl Zeiss Jena. Au cours des décennies qui suivent, cet organisme étatisé renoue avec la tradition industrieuse de la Thuringe, produit plus de 80 types de machines et investit en recherche et développement. Mais des difficultés surgissent pour l'approvisionnement et l'exportation. En 1970, la tension entre les firmes de l'Ouest et de l'Est atteint son paroxysme, chacune réclamant le droit d'utiliser la marque Zeiss. II s'ensuit de nombreux procès dont la décision finale est d'accorder la marque Zeiss ou Carl Zeiss uniquement aux produits manufacturés en Allemagne de l'Ouest à Oberkochen.

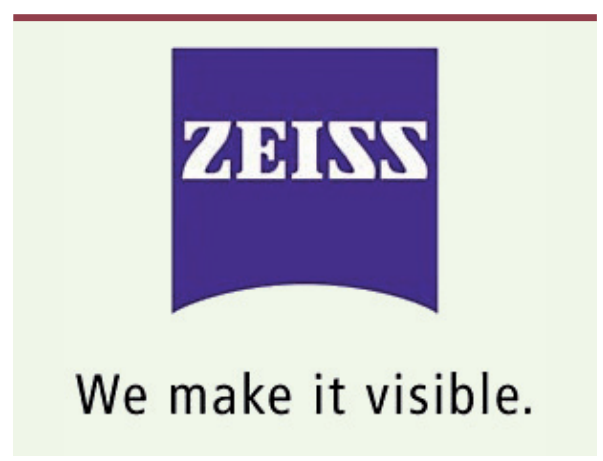

Logo depuis la réunification

\section{Réunification et épilogue}

Avec la chute du mur et l'effondrement du communisme, la réunification des deux groupes était prévisible. En 1990, Zeiss Oberkochen, avec ses 10200 employés et son excellent chiffre d'affaires, n'est pas disposée à acquérir Carl Zeiss léna, avec son personnel pléthorique et sa faible productivité. Des mesures radicales sont prises sous la direction de Lothar Späth (ex-ministre président du Land de Bade-Wurtemberg), devenu directeur de la partie essentielle de Carl Zeiss léna, désormais appelé Jenoptik Carl Zeiss Jena $\mathrm{GmbH}$. Elles sont radicales en effet : - reclassement d'une partie des effectifs ; 
- restructuration de l'ex-combinat et «assainissement » des bâtiments, transformés en zone industrielle pour des start up ;

- orientation de Jenoptik $\mathrm{GmbH}$ vers le laser, la micro-électronique et l'opto-électronique.

Ce traitement par Shock Therapy ${ }^{4}$ ne s'est pas fait, on l'imagine, sans douleur [6]. À l'un des 3000 employés de léna à qui on demandait comment s'était passée la restructuration, la réponse fut: «Ah oui, j'ai vu leur Mercédès dans le parking... ».

En dépit des 400 millions de dollars dépensés par l'Allemagne fédérale pour la réunification, les difficultés économiques accompagnant la transition d'une économie planifiée au système libéral ont d'abord entraîné une récession, et une « ostalgie » de la part des gens de l'Est.

Mais une étude du Centre d'information et de recherche sur l'Allemagne (CIRAC) de 2006 permet néanmoins de titrer «Comment l'innovation a résisté au Mur » [7].

Car, aujourd'hui, avec son effectif mondial de près de 14200 employés, son chiffre d'affaires de plus de deux milliards d'euros en 2000, l'entreprise possède des unités de production en Europe, en Amérique du Nord et au Mexique, ainsi qu'en Asie et continue son expansion, en particulier dans le domaine médical avec Carl-Zeiss-Meditec AG, résultat en 2002 de la fusion de la division ophtalmologie avec la firme Asclepion-Meditec AG. Désormais, la firme rend compte régulièrement de ses initiatives en recherche et développement dans un magazine accessible sur son site [8]. Malgré son comportement exemplaire pendant la période nazie, l'autre compagnie d'optique allemande, la maison Leitz, que nous aurons l'occasion d'évoquer dans un article ultérieur $(\rightarrow)$, ne connaîtra pas une telle réussite. $\diamond$

$(\rightarrow) \mathrm{m} / \mathrm{s}, 2011$ (sous presse)

\section{SUMMARY}

Greatness and tribulations of Zeiss and Leitz, two famous German optic companies. I. Zeiss

In a series of three articles, a parallel is drawn between the histories of the two most famous German optic companies, Zeiss and Leitz. Born in the middle of the 19th century, Zeiss went through National Socialism, World War II, partition and reunification of Germany. Archive documents abound, but a careful analysis is necessary to understand or guess the part played by the main actors, as Ernst Abbe, brilliant German physicist, or Küppenbender who eclipsed Emanuel Goldberg, a pioneer in information storage and retrieval. The reunification of Germany provided the opportunity for the reuniting of the eastern and western Carl Zeiss enterprises, creating a macro-economic shock, with radical change for the Carl Zeiss Jena company. Today, Carl Zeiss AG is a global leader in the optical and optoelectronic industry. $\diamond$

\section{CONFLIT D'INTÉRÊTS}

L'auteur déclare n'avoir aucun conflit d'intérêts concernant les données publiées dans cet article.

\section{RÉFÉRENCES}

1. Walker M. German national socialism and the quest for nuclear power 19391949. Cambridge : Cambridge University Press, 1993 : 302 p.

2. Goldberg $\varepsilon$. The densograph. BrJ Photography $1910 ; 57: 649-51$.

3. Goldberg $\varepsilon$. Die aufbau des photographisches bildes, $2^{e}$ ed. Halle : Knapp, 1925.

4. Goldberg $\varepsilon$. Das registrierproblem in der photographie. In : Herausg von J Eggert, A. von Bieler, eds. Bericht über den VIII internationalen kongress für wissenschaftliche und angewandte photographie, Dresden, 1931. Leipzig : J.A. Barth, 1932 : 317-20.

5. Buckland MK. Histories, heritages, and the past : the case of Emmanuel Goldberg. Conference on the history and heritage of scientific and technical information systems, Philadelphie, 15-17 novembre 2002.

6. Kogut $B$, Zander U. Did socialism fail to innovate? A natural experiment of the two Zeiss companies. Am Sociol Rev $2000 ; 65: 169-90$.

7. Guiot C. Carl Zeiss AG, ou : comment l'innovation a résisté au Mur. Regards sur l'économie allemande $2006 ; 79$ : 33-6 (en ligne : http://rea.revues.org/ index729.html).

8. http://www.zeiss.com/innovation

${ }^{4}$ La Shock Therapy (thérapie de choc) concerne la restructuration économique radicale d'un pays vers un système libéral, capitaliste et ouvert au commerce international.

\section{TIRÉS À PART}

S. Gilgenkrantz

\section{Bon de commande}

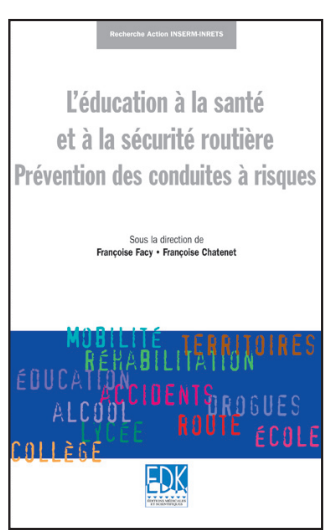

ISBN : 978-2-8425-4138-5 438 pages
À retourner à EDK, 2, rue Troyon - 92316 Sèvres Cedex

Tél. : 0155641393 - Fax : 0155641394 - E-mail : edk@edk.fr

NOM :

Prénom :

Adresse :

Code postal :

Ville :

Pays :

Fonction :

Je souhaite recevoir l'ouvrage L'éducation à la santé et à la sécurité routière : $20 €+3 €$ de port $=\mathbf{2 3} € \mathbf{T T C}$

en ................ exemplaire, soit un total de ..................................... €

$\square$ Par chèque, à l'ordre de $\mathbf{E} \mathbf{D} \mathbf{K}$

$\square$ Par carte bancaire : $\square$ Visa $\square$ Eurocard/Mastercard

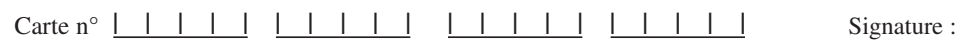

Date d'expiration: $\quad 1 \quad 1 \quad 1 \quad 1 \quad$

$\mathrm{N}^{\circ}$ de contrôle au dos de la carte : $\quad \underline{1} \mid$ 